\title{
Index
}

Note: Page numbers in italics refer to figures

Abdominal exploration 31-2

Abdominal pain 1,73

Abdominal palpation 73, 74, 88, 103, 106

Acepromazine 131

Acid blockers (anti-ulcer drugs)

$\mathrm{H}_{2}$ antagonists 134, 192

proton pump inhibitors (PPIs) 134-5, 165, 192

Acquired megaoesophagus 167-8

Activated charcoal 135

Acute colitis 226

Acute diarrhoea

diagnostic algorithm 83, 225

diagnostic tests $85-6$

differential diagnoses $84-5$

history 83

physical examination 83

treatment 140, 142

not indicated 141

zoonotic considerations 202

(see also Antidiarrhoeals; Chronic diarrhoea)

Acute gastritis 179, 186-7

Acute haemorrhagic diarrhoea syndrome (AHDS) 85 ,

204-5

and haematemesis 92,94

treatment not indicated 141

Acute hepatitis 255-7

Acute vomiting

clinical signs 72

diagnostic algorithm 72

diagnostic tests 74

differential diagnoses $71-2$

history 72

initiation of 71

physical examination $72-4$

signalment 72

treatment not indicated 140,141

(see also Antiemetics; Chronic vomiting)

Addison's disease see Hypoadrenocorticism

Adenocarcinoma 193-4, 229

Aerophagia 81

Algae colitis 228

Alpha ${ }_{1}$-proteinase inhibitor $\left(\alpha_{1}-\mathrm{PI}\right)$, faecal biomarker 5

Aminopenicillins 143

5-Aminosalicyclic acid derivatives 138

Amphimerus pseudofelineus 243

Ampicillin 274
Anaemia

cobalamin (vitamin $\mathrm{B}_{12}$ ) deficiency 119

hepatobiliary disease 248-9

sign of Gl disease 122

Anal furunculosis 103

Ancylostoma canium 9, 145

Ancylostoma tubaeforme 145

Ankyloglossia 152

Anorexia/hyporexia

diagnostic algorithm 50

diagnostic tests 51

differential diagnoses 49

history 49,50

physical examination 51

primary and secondary GI diseases 1

Antacids 133

Anthelmintics 146

Antibacterials 140-3

aminopenicillins 143

antibiotic resistance 140

fluoroquinolones 143

gastrointestinal conditions

in which they are indicated 140, 142

in which they are not indicated 140-1

in which they may not be indicated $140,141-2$

macrolides 143

nitroimidazoles 143

sulphonamides 143

tetracyclines 143

(see also individual drugs)

Antibiotic-responsive diarrhoea 142, 218-19

Antibiotics 217

resistance 140

(see also individual drugs)

Anticholinergics 131, 132, 136

Anticholinesterases 132

Antidiarrhoeals

antispasmodics 136

intestinal protectants and adsorbents 135

motility modifiers 135-6, 208

Antiemetics

centrally acting 130-1, 204, 207

peripherally acting 132

(see also individual drugs)

Antihistamines 131

Antimicrobials, gut microbiota manipulation 128

Antioxidants 129

Antispasmodics 136

Appetite stimulants 126, 139 
Arthropathies, sign of Gl disease 121

Ascarids (roundworms) 9, 84, 145, 209, 214

Ascites 80

diagnostic tests 111

diagnostic algorithm 112

differential diagnoses 111-12

effusion classification 109-10, 112

history 110

physical examination $110-11$

primary and secondary GI diseases 1

protein-losing enteropathy (PLE) 110

Atrophic gastritis 181, 189

Azathioprine 122, 138

Azotaemia, sign of Gl disease 120

Bacterial colitis 226-7

Bacterial diarrhoea 84, 209, 213

Bacterial oropharyngeal infection 154

Balantidium coli 8

Balsalazide 138

Basenji

breed-specific diseases 3

enteropathy 218

Bedlington Terrier, copper storage disease and copperassociated disease 253-4

Belgian Shepherd Dog

adenocarcinoma 193

breed-specific diseases 3

Bethanechol 132

Bile 244

Bile duct

neoplasia 262, 266

obstruction 265

rupture 266

Biliary stasis $246-7$

Biliary tract diseases $140,142,263-6$

causes $250-1$

diagnostic imaging 21

extra-hepatic biliary obstruction (EHBO) 265

feline chronic cholangitis 263-4

gall bladder mucocoele 264-5

neoplasia 262, 266

pathophysiology $245,246-7$

rupture 266

suppurative cholangitis/cholecystitis 263

Bilirubin encephalopathy, sign of Gl disease 120

Bilirubin metabolism 114

Biopsy 4, 40-5

diagnostic approach 4

endoscopic 29-30

future possibilities 45

gastrointestinal $32-3$

hepatic 33-5, 41, 250, 252, 254

muscle and peripheral nerve 67

pancreatic 35-6, 41

sample collection $40-1$

sample interpretation $44-5$

'virtual biopsy' 45

Bismuth salts 133, 135

Bloating

aetiology $80-1$

and ascites 80

diagnostic tests 81

differential diagnoses 80

history 81

pathophysiology 80-1

physical examination 81

primary and secondary GI diseases 1

treatment 81-2
Blood

complete count 94

digested, appearance 76, 92-3, 98

occult, faecal biomarker 5

testing 85-6, 90, 185, 248-9

(see also Haematemesis; Melaena)

Boxer, breed-specific diseases 3

Breed dispositions for specific diseases 3, 183

Budesonide 138

Bulk laxatives 136-7

Buprenorphine 207

Burns, oropharynx 156

Butorphanol 195, 208

Butylscopolamine 132

Calcinosis circumscripta 152, 153

Calgranulin/calprotectin, faecal biomarker 5

Campylobacter spp. 10, 140, 141, 227

Candida albicans 154

Canine chronic hepatitis

aetiology 251

breed dispositions 251

diagnosis 251-2

prognosis 253

treatment 252-3

Canine distemper virus 84, 153

Canine enteric coronavirus (CCoV) 84, 208-9

Canine familial hyperlipidaemia 260

Canine parvovirus (CPV) 84, 153, 206-8 and animal shelters 208

Capillaria putorii 180

Capromorelin 139

Cardiovascular signs of Gl disease 122

Cathartics see Laxatives and cathartics

Cestodes (tapeworms) 7, 8, 145, 209, 214-15 zoonotic considerations $214-15$

Chemical/caustic contact injuries, oropharynx 156

Chewing lesions 155

Chlorambucil 123, 138, 217, 222

Chlamydia 144

Chlorpromazine 131

Cholangitis/cholangiohepatitis 140, 142, 263-4

Cholestatic pruritus, sign of GI disease 121

Cholinergics 132

Chronic diarrhoea

clinical signs 88

diagnostic algorithm 89, 225

diagnostic tests 90

differential diagnoses $88-90$

history 87

physical examination 88

treatment 140

(see also Acute diarrhoea; Antidiarrhoeals)

Chronic enteropathies

antibacterials 142

breed-specific 218

dysautonomia 222-3

functional 222-3

inflammatory $215-18$

irritable bowel syndrome 222

neoplasia 219-20

protein-losing 220-22

small intestinal dysbiosis 218-19

visceral myopathy and chronic pseudo-obstruction 223

Chronic gastritis

atrophic 181, 189

causes 179-80

idiopathic 188-90 
infectious 187-8

ulcerative 180-1

Chronic hypertrophic pyloric gastropathy 190

Chronic inflammatory enteropathies

breed-specific 218

clinical presentation 215

diagnosis $215-17$

prognosis 217

severity assessment 218

treatment 217

Chronic vomiting

appetite changes 77

diagnostic algorithm 78

diagnostic tests 78-9

differential diagnosis $75-6,77$

history 76-8

physical examination 78

signalment $76-8$

(see also Acute vomiting; Antiemetics)

Ciclosporin 122, 138, 252

Cimetidine 134

Cisapride 132, 195

Cleft palate 151-2

Clostridium difficile 10, 140, 141, 226

Clostridium perfringens 10, 140, 141, 226

Co-amoxiclav 274

Cobalamin (vitamin $B_{12}$ ) 119, 128, 201, 202, 203

Coccidia 84, 214

Colestyramine 135

Colitis

acute 226

chronic 228

granulomatous 140, 142, 228

infectious 226-8

Collies, breed-specific diseases 3

Colon and rectum

abnormalities 224

anatomy 224

diagnostic algorithm 225

diagnostic imaging 13-19

diagnostic tests 224-6

diarrhoea 88, 225

diseases of

acute colitis 226

chronic colitis 228

constipation 225, 229-30

feline idiopathic megacolon 230

granulomatous colitis 140, 142, 228

infectious colitis 226-8

intussusception 230

irritable bowel syndrome (IBS) 230

neoplasia 229

obstipation 229-30

polyps 107, 229

endoscopy 25

function 224

physical examination 224

Colonic cleansers 137

Computed tomography (CT)

Gl tract 13, 19, 185, 193, 221

liver 22, 270

mandible 153

masticatory muscle myositis 157

pancreas 24

Congenital/developmental disorders

megaoesophagus 166-7

oropharynx 151-3

portosystemic shunts 269-72
Constipation 229-30

diagnostic algorithm 225

primary and secondary Gl diseases 1

Contrast radiography $3,13,16,70,166,184,191-2,194$, 211

Copper storage disease $253-5$

Coronavirus 84, 208-9, 228

Corticosteroids 138 (see also individual drugs)

Craniomandibular osteopathy (CMO) 152, 153

Cricopharyngeal achalasia 159

Cricopharyngeal asynchrony 159

Critical care diets 126

Cryptosporidium spp. 8, 214

Cutaneous abnormalities, sign of Gl disease 121

Cylicospirura felineus 180

Cyproheptadine 139

Cystoisospora spp. 8, 84, 214

Cytology 4, 40-5

Dantron (danthron) 137

Dehydration, fluid therapy $124-5$

Dental problems 61-3

chewing lesions 155

cleft palate 151-2

feline plasma cell gingivitis $154-5$

gingivostomatitis 154

necrotizing ulcerative gingivitis 154

periodontitis 153

temporomandibular joint (TMJ) disorders 152

Dental radiography 62

Diagnostic approach

biochemistry 3

biopsy $4,40-5$

breed-specific diseases 3, 183

cytology 4, 40-5

faecal examination 3, 5-11

haematology 3

history 3

imaging 3, 12-24

physical examination 3

specific tests 3-4

(see also individual conditions)

Diarrhoea 201

acute 83-6, 92, 94, 204-5, 225

bacterial $84,209,213$

causes 198

chronic 87-91, 225

primary and secondary GI diseases 1

treatment 135-6, 140, 142

not indicated 141

zoonotic considerations 202

Diazepam 139, 195, 257

Diet-induced acute enteritis 204

Diet-related acute diarrhoea 84

Diets see Nutrition

Digested blood ('coffee grounds')

chronic vomiting 76

haematemesis $92-3$

melaena 98

Digital rectal examination $88,103,107,224-6$

Diphenoxylate 136

Dipylidium canium 8, 145

Docusate sodium 137

Domperidone 132

Drooling

aetiology 56

diagnostic tests 58-9 
differential diagnoses 56,57

history 57

pathogenesis 56

physical examination $57-8$

primary and secondary Gl diseases 2

and rabies $57,58,59$

signalment 56

treatment 59

zoonotic considerations 59

Drug therapy (non-specific)

acid blockers (anti-ulcer drugs) 134-5

antidiarrhoeals 135-6

antiemetics 130-2

appetite stimulants 126,139

immunosuppressive/anti-inflammatory agents 138-9

laxatives and cathartics 136-7

mucosal (cyto)protectants 133-4

prokinetics $132-3$

(see also individual drugs)

Drug toxicity

and acute diarrhoea 84,85

and drooling 57

and hepatitis 256, 257

and jaundice 115, 117

and pancreatitis 233

Dysautonomia 183, 195, 222-3

oesophagus 170

Dyschezia

causes 103

clinical features 102

diagnostic algorithm 102

diagnostic tests 103-4

history 103

physical examination 103

Dysphagia

causes $64-5$

clinical signs $64-5$

diagnostic algorithm 66

diagnostic tests $65-7$

differential diagnoses 64

history 65

oesophageal 65

oral 64

pathogenesis $64-5$

pharyngeal 64-5

physical examination 65

primary and secondary GI diseases 2

signalment 65

treatment 67

(see also Swallowing)

Echinococcus spp. 8, 145

Effusions see Ascites

Electrical burns, oropharynx 156

Electrolyte abnormalities, sign of Gl disease 120

Electrolytes, fluid therapy 124

Electron microscopy 11, 42

Emollient laxatives 137

Endoparasitic infection 84

Endoscopy

biopsy 29-30, 40, 44, 180, 250

colonoscopy 28,100

cleaners 137

enemas 137

equipment $25-6$

foreign body removal 26,187

gastroduodenoscopy 94,97

gastroduodeno-colonoscopy 90

indications 25 laparoscopy 30,250

lower GI tract 27-8, 90, 100, 104, 107

normal appearance

lower Gl tract 29

upper Gl tract 28-9

oesophagus $67,70,162,164,165,168,171,172,173$, 174

proctoscopy $28,100,104,107$

small intestine 198, 211-12, 216

specialized techniques 30

stomach $181,182,186,188,190,191,192,193$

and ultrasonography 19

upper GI tract $26-7,67,70,94,97,162,164,165,168$,

$171,172,173,174,181,182,186,188,190,191,192$, 193, 198, 211-12, 216

Enemas 136,137

Enteral diets 126

Enteritis

acute haemorrhagic diarrhoea syndrome (AHDS) 85, 204-5

diet-induced acute enteritis 204

infectious enteritis 205-10

Enteropathogenic bacteria

antibacterials 141

Campylobacter spp. 10, 140, 141, 227

Clostridium spp. 10, 140, 141, 226

Escherichia coli 141, 227

faecal culture 10

faecal examination 10

Helicobacter spp. 140, 141, 179, 187

molecular fingerprinting 11

polymerase chain reaction 11

Salmonella spp. 10, 140, 141, 227

toxin assay 11

Eosinophilic gastritis 189

Eosinophilic stomatitis 154

EPI see Exocrine pancreatic insufficiency

Epithelial tumours 182

Erythromycin 132-3, 143

Escherichia coli 141, 227

Esomeprazole 135

Eurytrema procyonis 242

Exclusion diets 127

Exocrine pancreas

abscess 236

anatomy 231-2

biochemistry 231-2

biopsy 35-6, 41

diagnostic imaging 22-4

encephalopathy 119-20

exocrine pancreatic insufficiency (EPI) 236-41

and insulinoma 37-8

neoplasia 241-2

nodular hypoplasia 232

pancreatic bladder 243

pancreatitis $232-6$

surgical treatment $38-9$

parasites 242-3

partial pancreatectomy 37

physiology 231-2

pseudocysts 236

secretory proteins 231

(see also Exocrine pancreatic insufficiency; Pancreatitis)

Exocrine pancreatic insufficiency (EPI)

aetiology

chronic pancreatitis 237

hypoplasia/aplasia and congenital abnormalities 237 
miscellaneous 237

pancreatic acinar atrophy (PAA) 236, 237

clinical signs 238

diagnostic tests $238-9$

history 238

pathophysiology 237-8

and polyphagia 48

prognosis 241

treatment

antibiotic therapy 241

dietary modification 240

enzyme replacement 240

enzyme supplementation 240

glucocorticoid therapy 241

vitamin supplementation 240-1

(see also Exocrine pancreas; Pancreatitis)

Extra-hepatic biliary obstruction (EHBO) 265

Faecal elastase measurement, faecal biomarker 6

Faecal examination 85

diagnostic approach 3

enteropathogenic bacteria

Campylobacter spp. 10

Clostridium spp. 10

culture 10

molecular fingerprinting 11

polymerase chain reaction 11

Salmonella spp. 10

smear 10

toxin assay 11

faecal biomarkers

alpha ${ }_{1}$-proteinase inhibitor $\left(\alpha_{1}-\mathrm{Pl}\right) 5$

calgranulin/calprotectin 5

faecal elastase measurement 6

immunoglobulin A 5

lactoferrin 6

occult blood 5

parasites

antigen detection 7,10

concentration methods $6-7,8-9$

culture 7-10

immunofluorescence 7

polymerase chain reaction 10

smear 6

wet preparations 6

viruses

electron microscopy 11

immunological identification 11

polymerase chain reaction 11

zoonotic considerations 5,6

Faecal microbiota transplantation (FMT) 129

Familial PLE and PLN in Soft Coated Wheaten Terriers 218

Famotidine 134

Feeding techniques 126

Feline acquired pyloric stenosis 190

Feline calicivirus 153

Feline chronic cholangitis 263-4

Feline enteric coronavirus (FECoV) 84, 209, 228

Feline gastric actinomycosis 187-8

Feline gastrointestinal eosinophilic sclerosing fibroplasia 189

Feline hepatic amyloidosis 261

Feline hepatic lipidosis (FHL) 257-9

Feline herpesvirus 153

Feline idiopathic megacolon 230

Feline infectious peritonitis 228

Feline orofacial pain syndrome (FOPS) 160

Feline panleukopenia virus (FPV) 206-8

and animal shelters 208
Feline plasma cell gingivitis $154-5$

Fenbendazole 147, 210, 213, 242

Fibre, nutrition 127-8

Fluid therapy $124-5$

Fluoroquinolones 143

Folic acid (vitamin $\mathrm{B}_{9}$ ) 128, 202, 203

Food-associated skin disease, sign of Gl disease 121

French Bulldog, breed-specific diseases 3

Fungal colitis 227

Fungal gastritis 188

\section{Gall bladder}

biliary stasis $246-7$

biliary tract neoplasia 262, 266

biliary tract rupture 266

extra-hepatic biliary obstruction (EHBO) 265

diagnostic imaging 20,21, 249

feline chronic cholangitis 263-4

mucocoele 21, 250, 264-5

structure and function 244

suppurative cholangitis/cholecystitis 263

Gastric acid 178

Gastric dilatation-volvulus (GDV) 183, 195-6

Gastric disorders

acute gastritis $186-7$

chronic gastritis 187-90

chronic gastropathies 190

clinical signs 184

delayed emptying 194-6

diagnostic imaging 184-6

endoscopy 186

foreign bodies $183,190-1$

history 184

laboratory tests 184, 185

laparotomy 41, 186

motility disorders 194-6

neoplasia 193-4

physical examination 184

Gastric emptying 186

delayed 194-6

Gastric epithelial dysplasia 181

Gastric foreign bodies 183, 190-1

Gastric function evaluation 178, 186

Gastric hyperplasia 181-2

Gastric metaplasia 181-2

Gastric motility 178 disorders 183, 194-6

Gastric mucosal hypertrophy 181

Gastric neoplasia 182

Gastric outflow obstruction 182-3

Gastric parasites 180

Gastric perforation 181, 184, 185, 191

Gastric ulceration 93-4, 180-1, 191-2

Gastritis

acute see Acute gastritis

chronic see Chronic gastritis

Gastroduodenoscopy 94, 97

Gastrointestinal biopsy 32-3, 40-1

Gastrointestinal diseases

diagnostic approach $3-4$ biopsy $32-3,40-5,186$

cytology $40-5$

faecal examination $5-11$

imaging 12-24

presenting signs 1-2

Gastrointestinal immune system 199

Gastrointestinal pseudo-obstruction 183, 194 


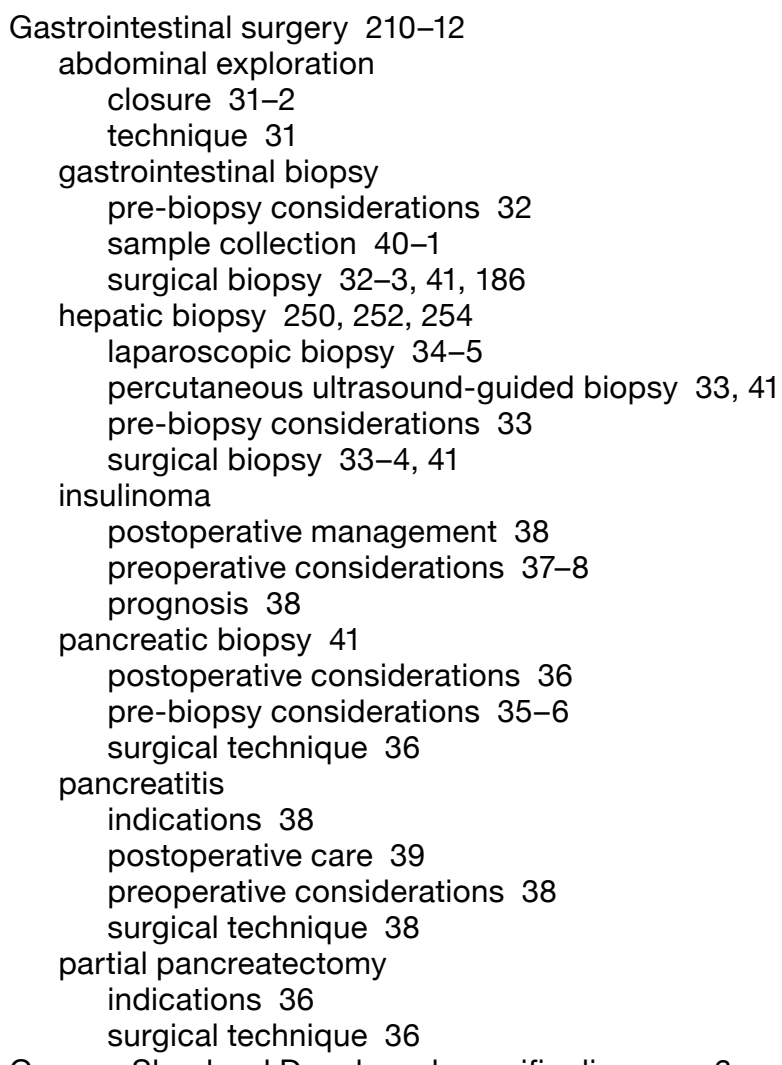

German Shepherd Dog, breed-specific diseases 3

Gastrointestinal tract

computed tomography 13, 19, 185, 193, 221

radiography $12-13,13-16,184-5$

ultrasonography 16-19, 186

Gastroscopy 181, 182, 186, 188, 190, 191, 192, 193

Giant hypertrophic gastritis (GHG) 189-90

Giardia spp.

and acute diarrhoea 84

detection 8,10

and small intestinal disease 213-14

Gingivostomatitis 62,154

Glucocorticoids 122, 222

Gluten-sensitive enteropathy (GSE) in Irish Setters 218

Glycogen-like vacuolar hepatopathy 259-60

Gnathostoma spinigerum 180

Granulomatous colitis 140, 142, 228

Gut microbiota 178

manipulation

antimicrobials 128

faecal microbiota transplantation (FMT) 129

prebiotics 128

probiotics 128-9

Gut-associated lymphoid tissue (GALT) 199

$\mathrm{H}_{2}$ antagonists 132, 134, 192

Haematemesis

diagnostic algorithm 93

diagnostic tests $93-4$

differential diagnoses 94

history $92-3$

physical examination 93

primary and secondary Gl diseases 2

Haematochezia

diagnostic algorithm 100

diagnostic tests 100

differential diagnoses 100

history 99

physical examination 99-100

primary and secondary Gl diseases 2
Haematology 3

Haemopoietic abnormalities in GI disease 122

Haemopoietic tumours 182

Halitosis

acute vomiting 73

diagnostic tests 62

differential diagnoses 60-1

history 61

pathophysiology 60

physical examination 61-2

primary and secondary GI diseases 2

signalment 61

treatment 63

Heavy metal ingestion, acute diarrhoea 84,85

Heinz body anaemia 115

Helicobacter spp. 140, 141, 179, 187

Helminths

anthelmintic agents 146

ascarids (roundworms) 9, 84, 145, 209, 214

cestodes (tapeworms) 7, 8, 145, 209, 214-15

colon 226

hookworms 9, 145, 209, 214

infectious enteritis 209

nematodes 145, 226

small intestine 209, 214-15

trematodes 226

whipworms 7, 9, 145, 209

zoonotic considerations 214-15

Hepatic arteriovenous malformation (HAVM) 275

Hepatic biopsy 33-5, 41, 250, 252, 254

Hepatic encephalopathy (HE) 119, 142, 268-70, 269, 273-4

Hepatic fibrosis 246

Hepatic lipidosis 257-9

Hepatitis 115,250

acute $255-7$

canine chronic 251-3

drug-induced 257

Hepatobiliary disease

acute hepatitis 255-7

biliary tract rupture 266

canine chronic hepatitis 251-3

causes $250-1$

copper storage disease and copper-associated disease

in cats 255

in dogs $253-5$

diagnosis tests $247-50$

drug-induced hepatitis 257

extra-hepatic biliary obstruction (EHBO) 265

feline chronic cholangitis 263-4

feline hepatic amyloidosis 261

feline hepatic lipidosis (FHL) 257-9

gall bladder mucocoele 264-5

neoplasia 261-3, 266

pathophysiology 245-7

superficial necrolytic dermatitis 121, 260-1

suppurative cholangitis/cholecystitis 263

vacuolar hepatopathies 259-60

Hepatocellular neoplasia 261-3

Hepatorenal syndrome, sign of GI disease 120-1

Hetastarch 125

Hiatal hernia 169-70, 185

Histiocytic ulcerative colitis see Granulomatous colitis

Histochemical stains, cytology 42

Histopathology, cytology 41-2

History, diagnostic approach 3

Hookworms 9, 145, 209, 214

Hyperammonaemic encephalopathies 119 
Hyperbilirubinaemia 114, 115, 121

Hypercoagulability secondary to PLE 120

Hypertriglyceridaemia 233

Hypertrophic gastritis 181, 189-90

Hypoadrenocorticism (Addison's disease) acute diarrhoea 84,85 haematemesis 94

Hypoalbuminaemia 120 fluid therapy 125

Hypocalcaemia 120

Hypoglycaemia 120

Hypokalaemia 120

Hyponatraemia 120

Hyporexia see Anorexia/hyporexia

Icterus see Jaundice

Idiopathic chronic colitis (ICC) 228

Idiopathic chronic gastritis 188-90

Idiopathic gastric motility disorders 194

Idiopathic non-inflammatory gastropathies 190

Imaging

acute diarrhoea 86

diagnostic approach 3

GI tract 12-19, 184-6

intestinal obstruction $210-11$

liver 19-22

pancreas 22-4

(see also individual techniques)

Immune system, gastrointestinal 199

Immune-mediated haemolytic anaemia 114, 115, 247

Immune-mediated disease

and drooling 57

haemolytic anaemia 114, 115, 247

oropharynx 154-5

Immunoglobulin A, faecal biomarker 5

Immunohistochemistry 43

Immunosuppressive/anti-inflammatory agents 138-9 (see also individual drugs)

Infectious diarrhoea 84

Infectious chronic gastritis 187-8

Infectious colitis 226-8

Infectious enteritis 205-10

Inflammatory hepatocellular disease 251-7

Insulinoma $37-8$

Intestinal obstruction 210-12

Intestine see Colon and rectum; Gastrointestinal biopsy; Gastrointestinal surgery; Small intestine

Intussusception 84, 85, 210, 211, 230

Irish Setters

breed-specific diseases 3

gluten-sensitive enteropathy (GSE) 218

Irritable bowel syndrome (IBS) 222, 230

Isospora see Cystoisospora

Itraconazole 188

Jaundice 78

bilirubin metabolism 114

classification 114

hepatic 115-16

post-hepatic 116

pre-hepatic 115

diagnostic algorithm 117

diagnostic tests 117

differential diagnosis 118

history 116

non-hepatic hyperbilirubinaemia 114

physical examination 116-17

treatment 118
Kaolin 135

Kernicterus see Bilirubin encephalopathy

Lactoferrin, faecal biomarker 6

Lactulose 137, 274

Large intestine see Colon and rectum

Laxatives and cathartics 136-7

Leucopenia 122

Liver

biliary tract diseases $263-6$

diagnostic imaging 19-22

differences between cats and dogs 245

hepatobillary diseases

classification 250-1

diagnosis $247-50$

hepatitis 250, 251-3, 255-7, 257

inflammatory 251-7

non-inflammatory 257-63

pathophysiology 245-7

structure and function 244-5, 268

vascular disorders

congenital portosystemic shunt (CPSS) 269-72

pathophysiology 268-9

hepatic arteriovenous malformation (HAVM) 275

hepatic encephalopathy (HE) 119, 268-70, 269, 273-4

peliosis hepatis $275-6$

portal vein hypoplasia $(\mathrm{PVH})$ 274-5

(see also Biliary tract diseases; Hepatic; Hepatitis; Hepatobiliary disease)

Loperamide 136

Low-fat/GI diets 127

Lymphangiectasia 3, 18, 47, 90, 110, 112, 127, 138, 201, $216,218,220-2$

Lymphoma 194, 220, 229

Lymphoplasmacytic gastritis $188-9$

Macroglossia 152

Macrolides 143

Malabsorption 201-2

Maropitant 130-1, 204, 207

Masticatory muscle myositis (MMM) 157-8

Megaoesophagus

acquired 167-8

aetiology 166

congenital 166-7

diagnosis 166-8

prognosis 169

treatment 168-9

Melaena

diagnostic algorithm 97

diagnostic tests $96-7$

differential diagnoses 98

history 96

physical examination 96

primary and secondary Gl diseases 2

small intestine 202

Ménétrier's disease-like gastritis 181, 182

Menrath's ulcer 157

Mesalazine 138

Mesenchymal tumours 182

Methadone 207

Metoclopramide 123, 131, 132, 195, 204, 207

Metronidazole 123, 143, 213, 219, 274

Microbiome, small intestine 199

Microbiota see Gut microbiota

Miniature Schnauzer, breed-specific diseases 3

Mirtazapine 139 
Mouth and throat see Oral cavity; Oropharynx; Salivary glands; Swallowing

Mucocutaneous diseases, oropharynx 154

Mucosal (cyto)protectants 133-4

Mucous metaplasia 181

Mumps, zoonotic considerations 161

Muscle dysfunction, sign of Gl disease 121

Musculoskeletal abnormalities of Gl disease 121

Myasthenia gravis 159

Mycophenolate mofetil 139

Mycoplasma 143

Necrotizing ulcerative gingivitis 154

Nematodes 145, 226

Neomycin 274

Neoplasia

and acute diarrhoea 84,85

adenocarcinoma 193-4, 229

biliary tract 262,266

hepatocellular 261-3

large intestine 229

lymphoma 194, 220, 229

oesophageal 173

oropharynx 156-7

salivary gland 161

small intestine 219-20

stomach $182,193-4$

Neurological signs of GI disease 119-20

Neuromuscular disorders, oropharynx 157-8

Nitroimidazoles 143

Nizatidine 134

Non-neoplastic polyps, gastric disease 190

Non-pharmacological therapies

fluid therapy 124-5

gut microbiota manipulation $128-9$

nutrition 125-8

other strategies 129

Non-steroidal anti-inflammatory drugs 138 (see also individual drugs)

Norwegian Lundehund breed-specific diseases 3 protein-losing enteropathy (PLE) 218

Nutrition 125-8

and acute diarrhoea 84

diets for acute Gl disease 127

enteral diets 126

exclusion diets 127

and exocrine pancreatic insufficiency 240

feeding techniques 126

fibre 127-8

low-fat/Gl diets 127

vitamins 128

Obstipation 229-30

Occult blood, faecal biomarker 5

Ocular abnormalities, signs of GI disease 122

Oesophageal diverticula 170-1

Oesophageal dysphagia 65

Oesophageal fistula 165-6

Oesophageal foreign bodies 173-5

Oesophageal neoplasia 173

Oesophageal stricture 171-3

Oesophagitis 163-5

Oesophagus

diagnostic imaging 12-13, 70, 163

dysphagia 65

endoscopy $25,26-7,67,70$

history 163

inflammatory disorders 163-6 laboratory tests 163

motility disorders 166-71

obstruction 171-5

pathophysiology 162

physical examination 163

structure and function 162

Ollulanus tricuspis 9, 180

Olsalazine 138

Omeprazole 134-5, 165, 174, 192, 207

Ondansetron 131, 207

Oomycetes, infectious colitis 227-8

Opioids 136, 207-8

(see also individual drugs)

Oral bleeding disorders 157

Oral cavity

diagnostic imaging 151

electromyography 151

histopathology 151

history 150

laboratory tests 151

pathophysiology 149-50

physical examination 150-1

structure and function 148-9

(see also Oropharynx; Salivary glands)

Oral dysphagia 64

Oral foreign bodies 155-6

Oral papilloma virus 153-4

Oronasal fistulae 61

Oropharynx

bleeding 157

congenital/developmental disorders 151-3

diagnostic imaging 151

direct injury 155-6

electromyography 151

functional disorders 158-60

histopathology 151

history 150

immune-mediated diseases 154-5

infectious/inflammatory diseases 153-4

laboratory tests 151

neoplasia 156-7

neuromuscular disorders $157-8$

pathophysiology 149-50

periodontitis 153

structure and function 148-9

(see also Oral cavity; Salivary glands)

Osmotic laxatives 137

Oxidative stress, supplemental antioxidants 129

Oxymorphone 195

Oxytetracycline 219

Pancreas see Exocrine pancreas

Pancreatectomy, partial 37

Pancreatic abscess 236

Pancreatic biopsy 35-6, 41

Pancreatic bladder 243

Pancreatic encephalopathy (PE) 119-20

Pancreatic pseudocysts 236

Pancreatitis

acute diarrhoea 85

acute pancreatitis 232

aetiology 232-3

chronic pancreatitis 232, 237

classification 232

diagnosis

diagnostic imaging 234

history and clinical signs 233

laboratory tests 234 
pathophysiology 232-3

prognosis 236

treatment 140,141

medical 234-6

surgical $38-9$

(see also Exocrine pancreas; Exocrine pancreatic insufficiency)

Pantoprazole 135, 207

Paraffin 137

Paraneoplastic effects, sign of Gl disease 121

Parasites 84

antigen detection 7,10

ascarids (roundworms) 9, 84, 145, 209, 214

cestodes (tapeworms) 7, 8, 145, 209, 214-15

colon 226

culture 7-10

egg size 7

faecal concentration methods $6-7,8-9$

faecal examination 6

gastric 180

hookworms 9, 145, 209, 214

immunodetection 7

nematodes 145, 226

pancreas $242-3$

polymerase chain reaction 10

small intestine 209-10, 213-15

treatment $145-7$

trematodes 226

whipworms 7, 9, 145, 209

zoonotic considerations $214-15$

Parasitic gastritis 188

Parasiticides 145-7

Pectin 135

Peliosis hepatis 275-6

D-penicillamine 255

Periodontitis 153

Pharyngeal dysphagia 64-5

Pharyngitis 154

Phenobarbital 131, 257

Phenothiazines 131

Physalopteria spp. 180

Phytochemicals, oropharynx 156

Poloxamers 137

Polymerase chain reaction 10,11

Polyphagia 46-8

diagnostic algorithm 46

diagnostic tests $47-8$

differential diagnoses 47

and exocrine pancreatic insufficiency (EPI) 48

history 46-7

physical examination 47

primary and secondary Gl diseases 2

Polyps

colon 229

non-neoplastic 190

rectal 107

Polyuria/polydipsia, sign of Gl disease 120

Portal hypertension 246

Portal vein hypoplasia (PVH) $274-5$

Portosystemic shunts 269-72

Postural (elevated) feeding 126

Prebiotics 128

Prednisolone 138, 217, 222, 236

Probiotics 128-9

Processionary moth caterpillars, oropharynx 156

Prochlorperazine 131

Prokinetics 132-3

Prostaglandins 133
Protein-losing enteropathy (PLE)

ascites 110

clinical presentation 221

complications 222

diagnosis 221

diseases causing 220-1

and hypercoagulability 120

Norwegian Lundehund 218

and pulmonary thromboembolism 122

small intestine 202

Soft Coated Wheaten Terrier 218

treatment 222

Yorkshire Terrier 218

Proton pump inhibitors (PPIs) 134-5, 165, 192

and haematemesis 94

Prototheca zopfi 228

Protozoa

and acute diarrhoea 84

faecal examination 6,8

infectious colitis 227

infectious enteritis 209-10

small intestine 213-14

treatment 145-7

Ptyalism/pseudoptyalism see Drooling

Pulmonary thromboembolism 122

Pyloric stenosis 194

Pyogranulomatous stomatitis 154

Rabies

drooling 57, 58, 59

zoonotic considerations 59

Radiography

dental 62

dysphagia 67

Gl tract 12-13, 13-16, 184-5

intestinal obstruction $210-11$

liver 19-20

pancreas 22-3

Ranitidine 134, 192, 204

Regurgitation 121

diagnostic algorithm 69

diagnostic tests $69-70$

differential diagnoses 68

history 68-9

physical examination 69

primary and secondary Gl diseases 2

signalment 68

treatment 70

Renal/urinary signs of Gl disease 120-1

Respiratory signs of GI disease 121-2

Resting energy requirement (RER) 126

Rickettsia 143

Ronidazole 123

Rottweiler, breed-specific diseases 3

Roundworms (ascarids) 9, 84, 145, 209, 214

Rupture of the biliary tract 266

Saline purgatives 137

Salivary glands $148,149,150,160-1$ (see also Oral cavity; Oropharynx)

Salmonella spp. 10, 140, 141, 227

Scottish Terrier, glycogen-like vacuolar hepatopathy 259-60

Shar Pei, breed-specific diseases 3

Sialadenitis 161

Sialadenosis 160

Sialocoeles 160 
Small intestinal dysbiosis (SID) 218-19

Small intestine

acute disease

acute haemorrhagic diarrhoea syndrome (AHDS)

$$
\text { 85, 204-5 }
$$

bacterial enteritis 209

diet-induced enteritis 204

intussusception 210, 211

obstruction 210-12

parasitic enteritis 209-10

viral enteritis 206-9

ascarids (roundworms) 9, 84, 145, 209, 214

cestodes (tapeworms) 7, 8, 145, 209, 214-15

chronic disease 88

breed-specific enteropathies 218

dysautonomia 222-3

infectious diseases

bacteria 213

helminths $214-15$

protozoa $213-14$

see also individual organisms

inflammatory enteropathies 215-18

irritable bowel syndrome 222

neoplasia 219-20

protein-losing enteropathy (PLE) 202, 220-1

small intestinal dysbiosis (SID) 218-19

visceral myopathy and chronic pseudo-obstruction 223

clinical signs of disease 198

diagnostic approach to disease 202-3

diagnostic imaging 13-19

diarrhoea 198, 201

endoscopy 25

hookworms 9, 145, 209, 214

malabsorption 201-2

melaena 202

pathophysiology 201-2

protein-losing enteropathy (PLE) 202, 220-1

structure and function 199, 200

whipworms 7, 9, 145, 209

Soft Coated Wheaten Terrier

breed-specific diseases 3

familial PLE and PLN 218

Staffordshire Bull Terrier, breed-specific diseases 3

Stomach

acute gastritis $179,186-7$

chronic gastritis 179-81, 187-90

chronic hypertrophic pyloric gastropathy 190

clinical signs of disease 184

delayed emptying 194-6

diagnostic imaging 13-19, 184-6

diagnostic investigation of disorders 184-6

dilatation-volvulus $183,195-6$

dysautonomia 183, 195

endoscopy 186

foreign bodies 190-1

hiatal hernia 185

hyperplasia 181-2

metaplasia 181-2

microbiota 178

motility disorders $183,194-6$

mucosal barrier 178, 180

neoplasia 182, 193-4

non-neoplastic polyps 190

outflow obstruction 182-3

parasites 180

pathophysiology of disorders 179-84 perforation 181, 184, 185

pseudo-obstruction 183, 194

pyloric stenosis 194

structure and function $177-8$

ulceration 180-1, 191-2

Strongyloides spp. 9

Sucralfate 133-4, 174, 192

Sulfasalazine 138

Sulphonamides 143

Superficial necrolytic dermatitis $121,260-1$

Suppurative cholangitis and cholecystitis 263

Swallowing 13, 148-50, 151, 159

see also Dysphagia; Oral cavity; Oropharynx

Tachypnoea 121

Tacrolimus 139

Taenia spp. 8, 145

Tapeworms (cestodes) 7, 8, 145, 209, 214-15 zoonotic considerations $214-15$

Temporomandibular joint (TMJ) disorders 152 osteoarthritis and ankylosis $152-3$

Tenesmus

diagnostic algorithm 105

diagnostic tests $107-8$

history 106

physical examination $106-7$

primary and secondary $\mathrm{Gl}$ diseases 2

Terbinafine 188

Tetracyclines 143

Thermal burns, oropharynx 156

Thiamine (vitamin $\mathrm{B}_{1}$ ) deficiency 120

Thrombocytopenia 122

Toads, oropharynx 156

Tocopherol (vitamin E) 128

Tonsillitis 154

Torovirus 84

Toxascaris leonina 9, 145

Toxin ingestion, acute diarrhoea 84, 85

Toxocara canis 9,145

Tramadol 208

Transmission electron microscopy (TEM), cytology 42

Trauma, oropharynx 155

Trematodes 226

Trichuris vulpis 9, 84, 226

Trimethoprim/sulphonamide 143, 214

Tritrichomonas foetus $8,103-4$

Tylosin 143, 219

Ulceration 93-4, 180-1, 191-2

Ulcerative gastritis $180-1$

Ultrasonography 216

endoscopic 19

GI tract 16-19, 186

haematemesis $93-4$

hepatobiliary disease 249

intestinal obstruction 211

liver 20-2

melaena 97

pancreas $23-4$

Uncinaria 9, 145

Urobilinogen excretion 114

Vacuolar hepatopathies in dogs 259-60

Vascular ring anomalies, oesophagus 175

Videofluoroscopy $67,70,151,159$

Viral diarrhoea 84

Viral infections

canine distemper virus 84,153 
canine enteric coronavirus $84,208-9$

canine parvovirus $84,153,206-8$

feline calcivirus 153

feline enteric coronavirus 84, 209, 228

feline herpesvirus 153

feline panleukopenia virus $206-8$

identification 11

oral papilloma virus $153-4$

rabies 59

torovirus 84

Visceral myopathy and chronic intestinal pseudoobstruction 223

Vitamin A 128

Vitamin $\mathrm{B}_{1}$ (thiamine) 120

Vitamin $\mathrm{B}_{9}$ (folic acid) 128, 202, 203

Vitamin $\mathrm{B}_{12}$ (cobalamin) 119, 128, 201, 202, 203

Vitamin D 128

Vitamin E (tocopherol) 128

Vitamin K 128, 250

Vomiting 121

acute 71-4

antiemetics 130-2, 204, 207

chronic 75-9

fluid therapy $124-5$

primary and secondary Gl diseases 2
Weight loss

diagnostic algorithm 53

diagnostic tests $54-5$

differential diagnoses $52-3,55$

history 52-4

physical examination 54

primary and secondary GI diseases 2

Weight loss/gain, polyphagia 46-8

Whipworms 7, 9, 145, 209

Xerostomia 161

Yersinia enterocolitica 227

Yorkshire Terrier

breed-specific diseases 3

protein-losing enteropathy (PLE) 218

Zoonotic considerations

acute diarrhoea 202

bacteria 213, 227

cestodes (tapeworms) 214-15

faecal examination 5, 6

helminths 214

mumps 161

rabies 59 